\title{
Analysis of the influence of process complexity and employee competence on the effect of digital assistance in industrial assembly
}

\author{
Thimo Keller ${ }^{1} \oplus \cdot$ Magnus Behling $^{1} \cdot$ Christopher Stockinger $^{2} \cdot$ Joachim Metternich $^{1} \cdot$ Klaus Schützer $^{3}$
}

Received: 3 July 2020 / Accepted: 24 November 2020 / Published online: 23 December 2020

(c) The Author(s) 2020

\begin{abstract}
The diversification of products increases the complexity of production, which intensifies the demands placed on employees. Digital assistance is expected to improve employee performance and flexibility. This paper examines the impact of digital assistance on labor productivity (hourly output) and experience of use in dependence on process complexity and employee competence. Within the framework of an empirical study in the process learning factory CiP of the TU Darmstadt, the effect was quantified using the example of a typical assembly process. This paper presents the concept and the results of the study. After the classification of an assembly task, these results can be used to support the selection decision for a digital assistance system according to cost-benefit aspects.
\end{abstract}

Keywords Digital manufacturing system $\cdot$ Assembly(ing) Productivity $\cdot$ Experience of use

\section{Motivation and preparatory work}

At present, manufacturing companies are confronted with a multitude of challenges [1]. Increasing product variety and smaller batch sizes have to be managed, while quality, time and costs have to be competitive in international comparison. Particularly in assembly, the need for greater flexibility increases the pressure on employees to remain productive and competitive. This applies especially to highwage countries. Overall this leads to a more complex work environment for the employees. More variants and components lead to more decisions to be made by the executing employee. For this reason, better support with information to assist decision making is needed [2]. In the context of these developments, it is urgently necessary to offer the affected

Thimo Keller

t.keller@ptw.tu-darmstadt.de

1 Institute for Production Management, Technology and Machine Tools (PTW), Technische Universität Darmstadt, Otto-Berndt Str. 2, 64287 Darmstadt, Germany

2 Institute for Ergonomics and Human Factors, Technische Universität Darmstadt, Otto-Berndt-Straße 2, 64287 Darmstadt, Germany

3 Production Engineering, Federal University of ABC, Av. dos Estados, 5001 Santo André, Brazil employees better access to information, e.g. through digital assistance systems.

Companies have high expectations regarding the introduction of such systems, but are hindered by the high implementation expenses in terms of time, costs and organizational or process adjustments [3]. Furthermore, there is great uncertainty regarding the benefits of single assistance technologies [3].

Thus, research on the effect of digital assistance has so far concentrated mainly on the comparison of different communication media and thus addresses the question of which hardware implementation of digital assistance is most promising. Funk et al. [4] and Blattgerste et al. [5], for example, show that digital assistance does not have a beneficial effect on assembly time, but primarily has a positive effect on quality. In contrast, Uva et al. [6], Hou et al. [7] and Aehnelt [8] show that digital assistance has a positive effect on both assembly time and assembly quality compared to paperbased work instructions.

The question of the influence of qualification and experience of employees on the effect of digital assistance is addressed by Jeske et al. [9] and Watson et al. [10], who examine in particular training processes and the appropriate media. On the one hand, they conclude that informatically rich and less abstracted assembly information is well suited for training processes. On the other hand, the learning curves determined show that these effects converge 
with increasing practice. Experienced and qualified assembly employees do not benefit as much from digital assistance as less qualified employees.

Although it is possible to deduce influences on the effect of digital assistance from these studies, two further aspects need to be considered with regard to the state of research: On the one hand, the studies mentioned above consider simple digital implementations for the transmission of assembly information. More extensive forms of digital assistance, which are, for example, already implemented in learning factories are not used (see e.g. [11-13]). However, in particular less qualified assembly workers could benefit from these. On the other hand, the studies do not vary the work task. This is remarkable, since the complexity of work task is mentioned in the practiceoriented literature as the main motivation for the use of digital assistance (see $[14,15])$.

This results in two major fields of action. On the one hand, by quantifying the effects of digital assistance systems, their acceptance is to be promoted and in a further step, a frame of reference is required that serves companies as a support to introduce the appropriate digital assistance systems, depending on their individual situation and challenges. In order to start closing this research gap, Keller, Bayer, Bausch and Metternich [16] have developed an investigation model based on a systematic literature research, which describes the effects of digital assistance on production processes. In addition, various test scenarios were defined and a procedure was developed to verify the investigation model. This preparatory work serves as a basis for the following elaborations and will be presented in more detail in chapter 2. On the other hand, it should be noted that with the qualification of employees and the complexity of the work task, two major influencing variables on the effect of digital assistance have so far been analyzed little or insufficiently. This is the starting point of this article, which presents an empirical study that aims to quantify these effects.
In this paper the study carried out and the corresponding results for the benefit evaluation of digital assistance are presented. This study will test the hypothesis that complexity and competence have an influence on the positive effect of digital assistance. As a result, we expect to find measurable effects of digital assistance, which differ depending on the test scenario. The purpose is to identify the scenario with the greatest effect. This scenario will be selected to investigate the functional relationships between digital assistance and the observed effects in higher detail.

In this article:

- an existing research model is adapted so that selected key performance indicators (KPIs) can be measured and the formulated hypotheses can be tested in the laboratory study (Sect. 2)

- an experimental setup is presented (Sect. 3)

- the design of the study is described (Sect. 4)

- the study results are presented and evaluated (Sect. 5)

\section{Investigation model and adaptations}

To carry out the study, the above-mentioned investigation model and the test scenarios had to be adapted and suitable KPIs had to be derived. The modified model for the study is shown in Fig. 1. The model illustrates the hypothetical interdependencies (red arrows) between the functions of the assistance system, the effects on the employee and the KPIs, which can be aggregated to labor productivity as an overall measure.

\subsection{Modifications of the model}

In a previous publication, eight functions of digital assistance were defined [16]. To validate the fundamental suitability of our approach we use the following five functions

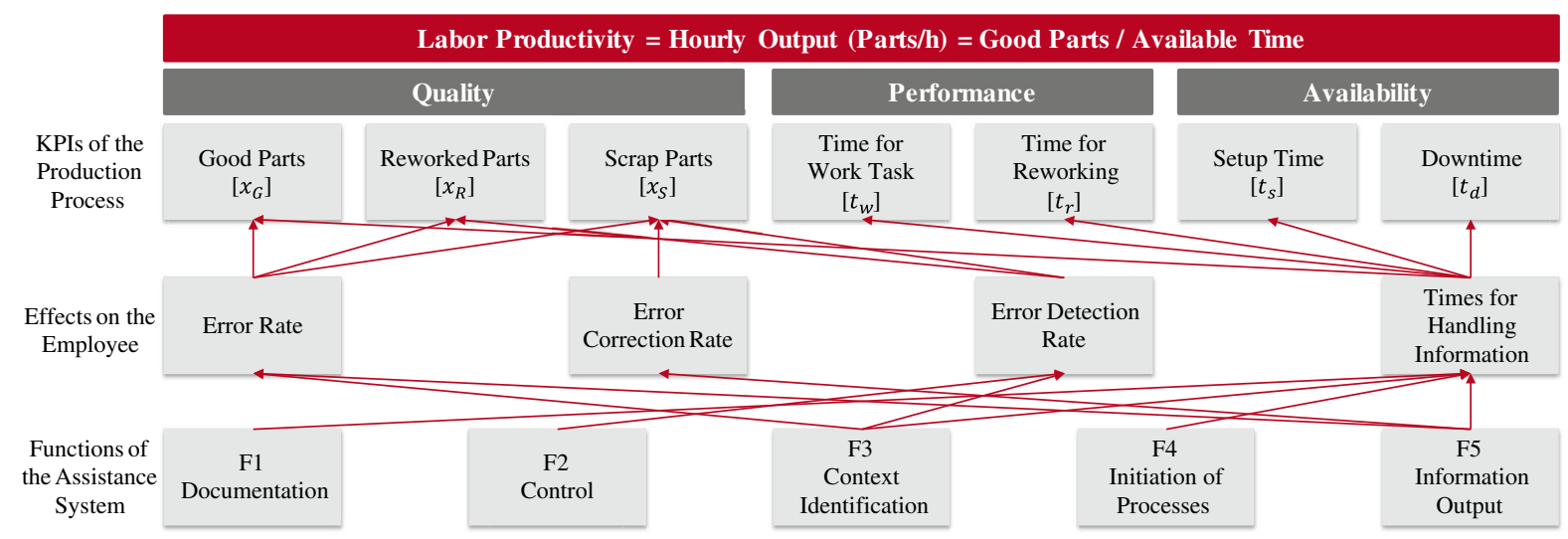

Fig. 1 Investigation model adapted to the study 
of digital assistance in this study: Documentation, Control, Context Identification, Initiation of the Process and Information Output.

Three further functions were not taken into account. The Decision Support, based on data analytics, as a function of an assistance system was not implemented, because at the time the study was conducted, there was not enough data available. Since the focus of the study is on the effect of digital assistance on the individual workplace, the function of Communication was not considered. Contrary to the focus of the study, this function describes the possibility of communicating with knowledge carriers or people in charge and thus is primarily focusing on network effects. Due to the study design, as it is described in Sect. 4, the function Learn and Practice was removed from the model. The design developed for the study does not provide for repetitions of the assembly process, but these are necessary to generate learning curves. Consequently, the effects on the employee and KPIs of the Production Process associated with the three removed functions have also been eliminated.

\subsection{Labor productivity as KPI to measure the benefit}

In order to measure the effect of digital assistance, a target variable is necessary. This variable must meet two requirements: It must be possible to causally attribute a change in this target variable to the use of digital assistance systems. In addition, it must be possible to measure or determine the components of the variable with reasonable effort for conducting the study and especially for a practicable application in a production process of a company. Furthermore, labor productivity is a common and widely used metric to measure the performance of work systems in production.

For manual assembly as a highly human-centred process, the definition of labor productivity as the ratio of output to labor input [17] is suitable. As a commonly used key figure in industrial production it enables the comparison of introducing digital assistance systems with other improvement measures in production. The operationalization of labor productivity (hourly output) is based on the Nakajima report on Overall Equipment Effectiveness (OEE) [18]. The factors availability, performance and quality are included in the calculation with one single term each, as it can be seen in Eq. 1. Since the assembly is carried out without repetitions, only time variables were used for calculation:

$L P=\frac{t-t_{d}-t_{s}}{t} \times \frac{t_{a}}{t-t_{d}-t_{s}} \times \frac{t_{w}}{t_{w}+t_{r}}$.

In Eq. $1 t$ represents the total time spend on the assembly line, $t_{d}$ describes the downtime $t_{s}$ the setup time, $t_{w}$ the time used for the work task, $t_{r}$ the time for reworking and $t_{a}$ is the target time for the assembly process of the product. This target time was determined for both products using the Methods-Time Measurement (MTM) method, which is a predetermined motion time system and does not contain any time for the handling of information, which means that the determined target time describes the absolute minimum time required for the work task [19]. The first term represents the ratio of available time to total time, the second the ratio of target time to required time (performance) and the third the proportion of working time in which work was carried out without errors (quality).

\subsection{Questionnaire meCUE as KPI to measure the user experience}

The success of digital assistance systems depends not only on economic considerations but also on the assessment of the employees who work directly with these systems. Because assembly is strongly human-centered, it is important that employees can and want to work effectively with digital assistance systems. For this reason, the experience of use is also recorded in addition to work productivity. This can be measured using the questionnaire meCUE [20]. This questionnaire comprises a total of 33 items in the categories task-related product perception, non-task related product perception, user emotions and consequences of use, which are recorded using a seven-level Likert scale.

\subsection{Categorisation of the test scenarios}

The categorisation of the test scenarios with regard to employee competence and process complexity is implemented as follows.

To address competency it was distinguished between trained and untrained test persons. In the study carried out, process-experienced test persons ("trained") received an introduction to the corresponding product before assembly, in which the product variant was assembled and disassembled twice under instruction. This is considered sufficient to gain adequate process knowledge. Untrained test persons have no knowledge of the product before assembly.

Process complexity was addressed by the use of two products, which are available in the process learning factory $\mathrm{CiP}$ of the TU Darmstadt: A pneumatic cylinder from Festo and a gear motor from SEW Eurodrive (see Fig. 2). These products, which serve as demonstrators, were used to conduct the study. After a detailed methods-time measurement (MTM), the assembly of the gear motor (Target time for sum of activities $=5.7 \mathrm{~min}$.) can be considered much more complex than the pneumatic cylinder (2.1 min.). This result corresponds to the classification into a low and a medium complex product according to the categorization of a previous publication of the authors [16]. 


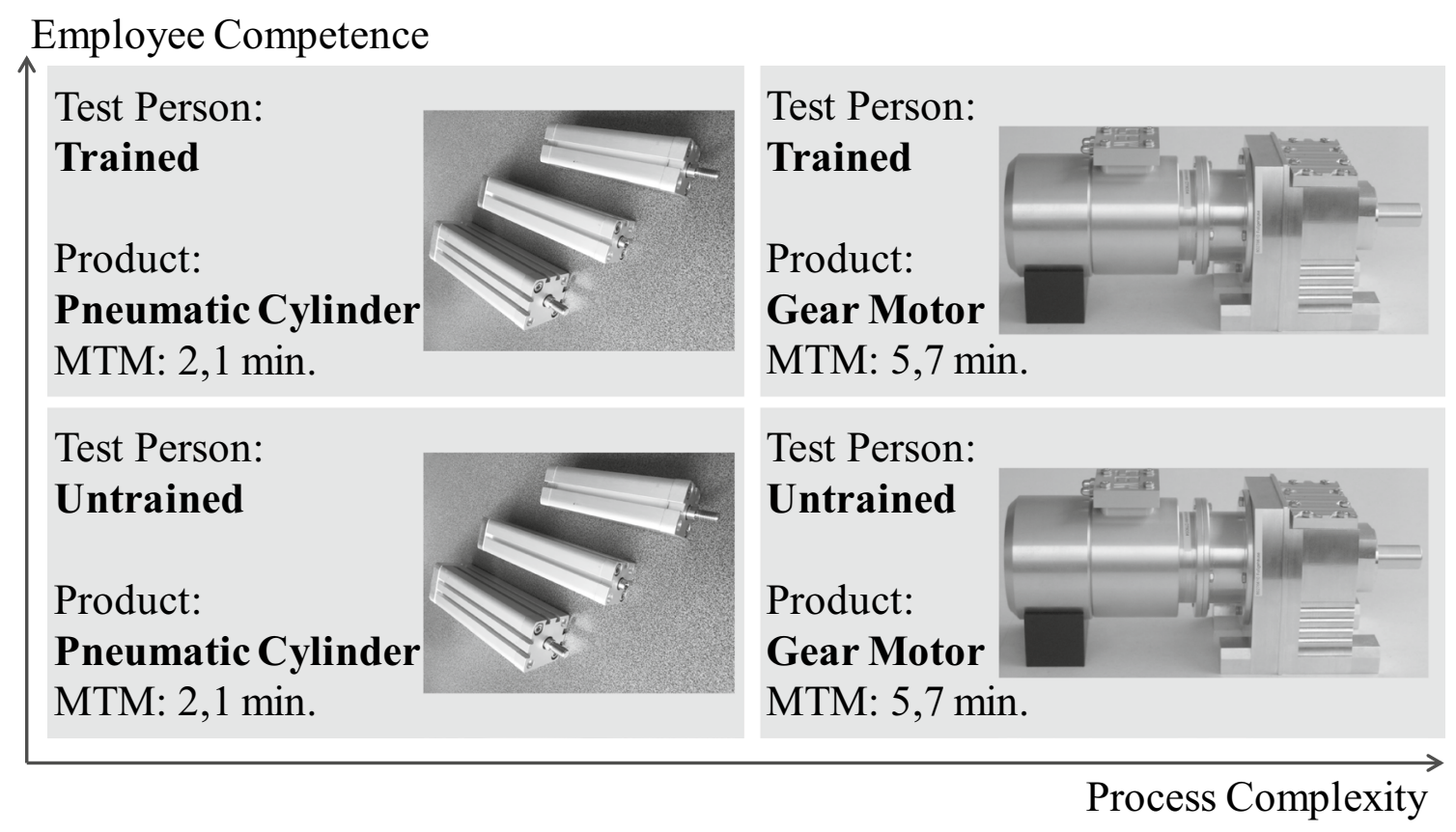

Fig. 2 Test scenarios for the conducted study

\section{Experimental setup in the process learning factory CiP}

Both products are assembled on the assembly line shown in Fig. 3 in the process learning factory CiP. This manual assembly line consists of three workstations which are passed through by the test person during the assembly of a product in sequence in a one-piece flow. The assembly line is equipped with pick by light technology, touch screen monitors, controlled assembly tools and other sensor technology. These components are interconnected via a process control system and can be controlled by this system during the assembly process. This setup allows the realization of all five functions mentioned in Sect. 2.1 (see Fig. 1). The functions are partly realized by a combination of individual elements of the technical setup. For example, the control is carried out by data acquisition via the sensor system and a comparison with the target value in the process control system.

\section{Study design}

The aim of the study is to investigate the effect of digital assistance depending on process complexity and employee competence. For this purpose, we must first test whether digital assistance has a significant positive effect on productivity:

$\mathrm{H} 1$ : It is assumed that the productivity of the assembly process is greater with digital assistance $\left(\mu_{a}\right)$ than without $\left(\mu_{w}\right)$. The hypotheses can be formulated as follows:
$H_{0}: \boldsymbol{\Delta}=\mu_{a}-\mu_{w} \leq 0$ and $H_{1}: \boldsymbol{\Delta}=\mu_{a}-\mu_{w}>0$.

Once the hypotheses formulated in Eq. 2 have been tested for each scenario, the respective effect size of digital assistance is compared between the scenarios via the Cohens $d$. On this basis the following assumptions can be verified:

- H1.1: The effect (size) of digital assistance on labor productivity is larger for the gear motor than for the pneumatic cylinder.

- H1.2: The effect (size) of digital assistance on labor productivity is larger for untrained test persons than for trained test persons.

It was also tested whether the digital assistance has a significant positive effect on the user experience:

$\mathrm{H} 2$ : It is assumed that the experience of use is greater with digital assistance $\left(\mu_{a}\right)$ than without $\left(\mu_{w}\right)$. The hypothesis can be formulated accordingly to Eq. 2. Cohens d is used to compare effect sizes between the scenarios for experience of use:

- H2.1: The effect (size) of digital assistance on experience of use is larger for the gear motor than for the pneumatic cylinder.

- H2.2: The effect (size) of digital assistance on experience of use is larger for untrained test persons than for trained test persons. 


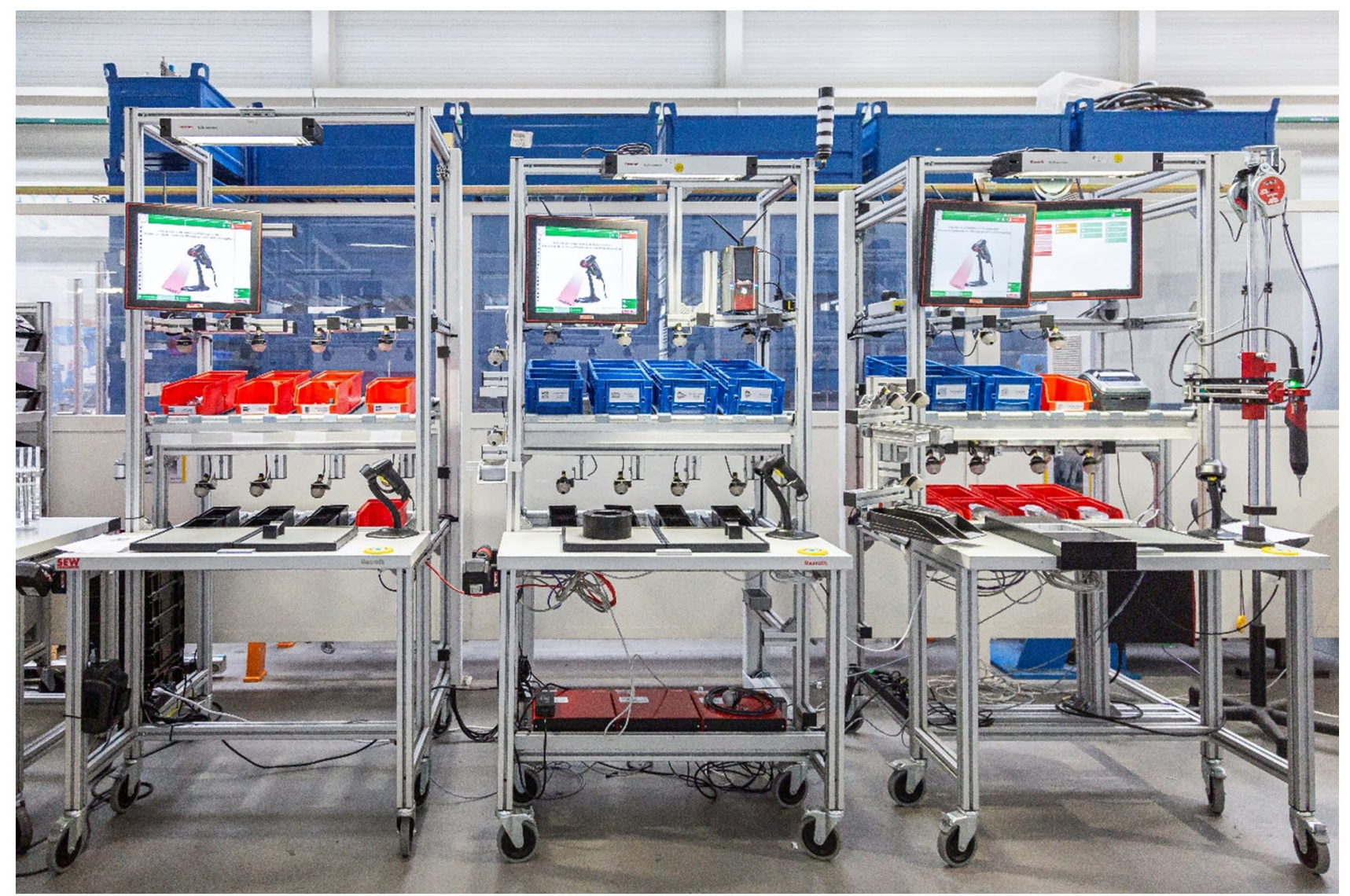

Fig. 3 Assembly line in the process learning factory $\mathrm{CiP}$

The within-subject design was chosen for the study, which means that all participants were exposed to several conditions. Each test person assembles both products once with and once without digital assistance. Thereby, each participant is trained for one product and untrained for the other. The assembly sequence was systematically varied to counterbalance order effects. One procedure contains three essential components:

1. Set up of the assembly line;

2. Assembly of the product;

3. If a product is assembled incorrectly, the error must be reworked afterwards.

When considering one test scenario separately, the mean values of labor productivity with and without digital assistance are compared by means of a paired $t$ test. The t-test is used to test the statistical significance of a difference in means. The comparison is one-sided.

The comparison between the test scenarios is based on the Cohens $d$ as a measure of the effect size and can be performed based on the t-tests. Compared to statistical significance, the consideration of effect size offers two advantages:
1. The informational value is greater, because in addition to the significance, the practical relevance (relative size of the effect) is also evaluated.

2. The comparability of results of different studies (in our case test scenarios) is simplified.

Cohen [21] provides a frame of reference for the interpretation. Thus $d=0.20$ corresponds to a small effect size, $d=0.50$ to a medium effect size and $d=0.80$ to a large effect size [21].

To determine the required number of participants, the expected effect size must be estimated. A very strong effect is anticipated when the complex product is assembled by an untrained test person. Since the effect is likely to be weaker for the remaining three test scenarios, a medium to large effect is assumed. This leads to the expected effect size of $\dot{d}_{3}=0.70$. Following the comments of Cohen [9] the required number of test persons (n) can be calculated:

$$
n=\frac{n_{10}}{100 \times\left(\dot{d}_{3} \times \sqrt{2}\right)^{2}}+0.7 .
$$

Choosing a conventional significance level of $\alpha=0.05$ and a statistical power of $\beta=0.80$, the table value $n_{10}$ 
contained in Eq. 3 can be determined and it follows that 30 test persons are required in total. Looking at it from the other perspective, significant results can be expected if the study is performed with at least 30 test persons.

\section{Study results and evaluation}

Thirty subjects ( 25 male/5 female) with an average age of 22.9 years participated in the study. Twenty-seven of them were students, most of them in technical subjects. None of them had any previous experience with the assembly line, but some had experience with the assembly of the pneumatic cylinder. These were placed in the group of subjects trained for the cylinder.

Figure 4 shows the mean difference in labor productivity $\Delta$, its improvement in relation to the averaged productivity without digital assistance, the Cohens $d$ (if the effect is statistically significant), the $t$-value and the $p$-value for each test scenario. The stars represent the statistical significance with zero stars corresponding to $\mathrm{p}>0.05$, one star corresponding to $0.01<\mathrm{p} \leq 0.05$ and two stars corresponding to $\mathrm{p} \leq 0.01$.

Regarding the hypotheses raised in Sect. 4 , the analysis of labor productivity reveals two main points:

- The effect size of digital assistance is greater for the gear motor than for the pneumatic cylinder

- The effect size of digital assistance is greater for untrained employees than for trained employees
Analysing the setup time separately, it is observed that digital assistance has a significantly negative effect on setup time for the gear motor $(\mathrm{t}(29)=-2963, \mathrm{p}=0.003)$ and for the pneumatic cylinder $(\mathrm{t}(27)=-1880, \mathrm{p}=0.036)$. The reason for this effect may be the simplicity of the setup process in terms of time and content. The digital assistance seems to unnecessarily bind the attention of the operator. Due to this effect, the lead time not including the setup process is examined and the following is observed:

- The relation between the test scenarios is identical to the previous analysis of labor productivity

- By removing setup time from the analysis, all observations are reinforced in the dimensions of significance and effect size

Figure 4 shows the mean difference of the meCUE results $\Delta$, its improvement in relation to the averaged experience of use without digital assistance, Cohens d, the $t$-value and the $p$-value for each test scenario. The stars represent the statistical significance as mentioned previously. Regarding the hypotheses raised in Sect. 4, the results of experience are consistent with those for work productivity:

- The effect size of digital assistance is greater for the gear motor than for the pneumatic cylinder

- The effect size of digital assistance is greater for untrained employees than for trained employees

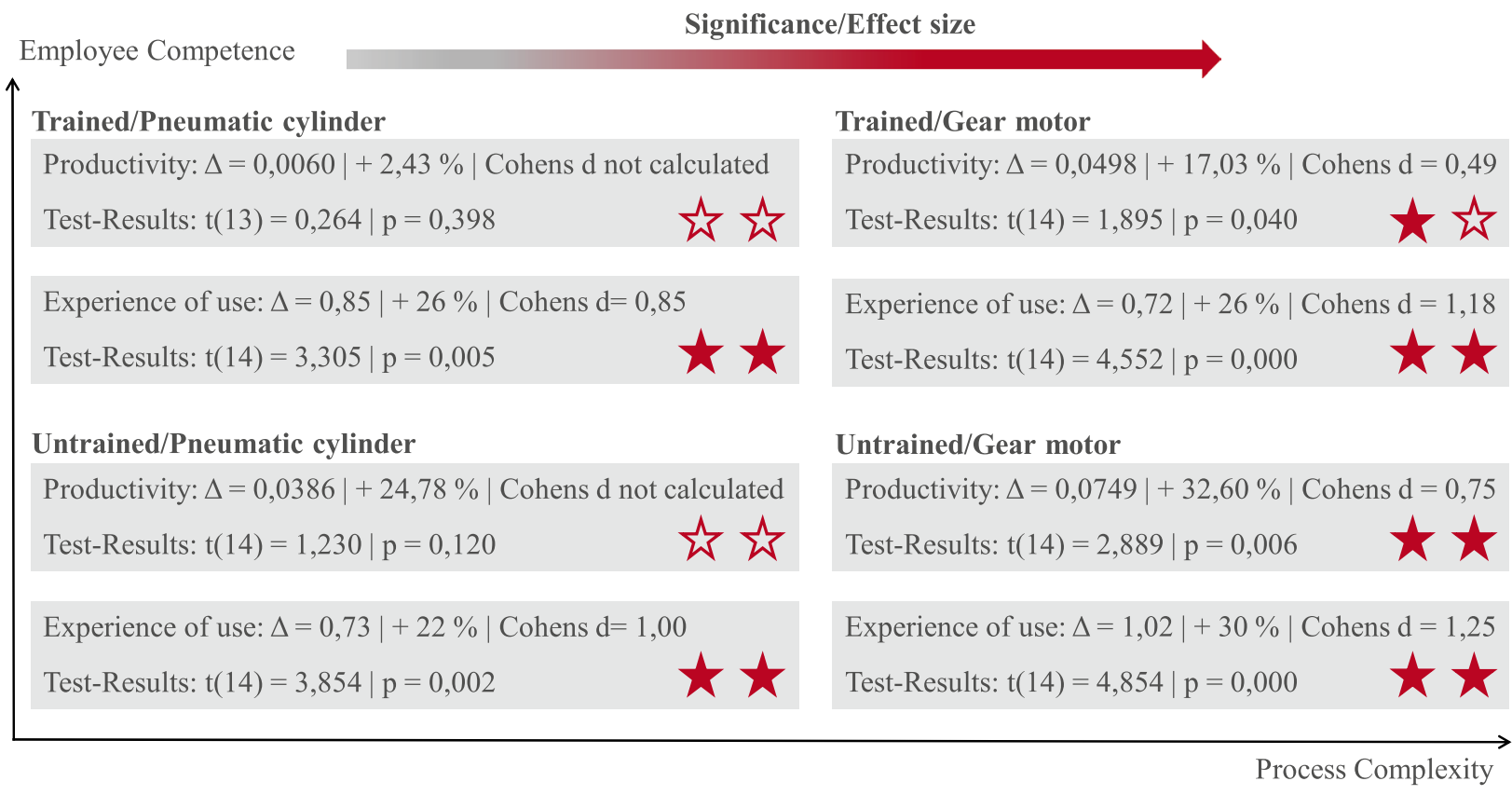

Fig. 4 Statistical Results of the analysis of labor productivity and user experience 
Thus, the hypotheses raised in Sect. 4 can be clearly confirmed. More generally, this study indicates that the effect of digital assistance increases with greater process complexity and decreases with higher employee competence.

From the study results the following transferable new knowledge can be derived: As shown the effect of digital assistance increases for more complex tasks. Taking the setup process into account, the rising benefit of digital assistance could be observed over three levels of complexity. In the same way, the increase of the positive effect with decreasing employee competence could be verified. These findings assist companies in identifying meaningful areas of application for digital assistance systems. The focus should be on rather complex assembly processes and less competent employees (e.g. working students). This shows, for example, that digital assistance is predestined to support training processes for workplaces with complex assembly tasks, especially if the employees have little knowledge of the (or a comparable) assembly process. Furthermore it could be observed that the experience of use was in every scenario better with digital assistance than without. This leads to the expectation that the acceptance for a (well implemented) digital assistance exists and even increases with higher usage (high complexity, low competence).

\section{Summary and outlook}

The aim of this study was to investigate the influence of process complexity and employee competence on the effect of digital assistance. Therefore, it was set up an assembly line with several functions of digital assistance in the process learning factory $\mathrm{CiP}$. First, the investigated target value of labor productivity and experience of use and various test scenarios were defined. In order to operationalize the categorization of test scenarios, practice-oriented criteria were introduced. The central result of the investigation is that the effect of digital assistance increases for the more complex product and untrained test persons, supporting [9] and [10]. If absolute values are considered, digital assistance always has a positive effect for the complex product. For simple products (cylinder) and simple activities (set up), it is shown that digital assistance has no significant or a negative effect.

Based on these results, the model shown in Fig. 1 will now be analysed in further studies. For the identified test scenario with the greatest effect size of digital assistance, the individual functions of the assistance system are isolated and systematically varied. In addition, the influence of the individual functions on the subordinate categories of Labor Productivity (Availability, Performance and Quality) is differentiated in following studies. Using multiple regression, the influences of the individual functions are then quantified to create a reference framework for the introduction of digital assistance, individually adapted to existing processes, as mentioned in the introduction.

Funding Open Access funding enabled and organized by Projekt DEAL. This research did not receive any specific grant from funding agencies in the public, commercial, or not-for-profit sectors.

\section{Compliance with ethical standards}

Conflict of interest The author declares that there is no competing interest.

Open Access This article is licensed under a Creative Commons Attribution 4.0 International License, which permits use, sharing, adaptation, distribution and reproduction in any medium or format, as long as you give appropriate credit to the original author(s) and the source, provide a link to the Creative Commons licence, and indicate if changes were made. The images or other third party material in this article are included in the article's Creative Commons licence, unless indicated otherwise in a credit line to the material. If material is not included in the article's Creative Commons licence and your intended use is not permitted by statutory regulation or exceeds the permitted use, you will need to obtain permission directly from the copyright holder. To view a copy of this licence, visit http://creativecommons.org/licenses/by/4.0/.

\section{References}

1. AlGeddawy T, ElMaraghy H (2012) Product variety management in design and manufacturing: challenges and strategies. In: ElMaraghy $\mathrm{H}$ (ed) Enabling manufacturing competitiveness and economic sustainability. Springer, Berlin, pp 518-523

2. Fast-Berglund Å, Fässberg T, Hellman F, Davidsson A, Stahre J (2013) Relations between complexity, quality and cognitive automation in mixed-model assembly. J Manuf Syst 32(3):449-455

3. Klapper J, Gelec E, Pokorni B, Hämmerle M, Rothenberger R (2019): Potenziale digitaler Assistenzsysteme. Aktueller und zukünftiger Einsatz digitaler Assistenzsysteme in produzierenden Unternehmen. Fraunhofer-Institut für Arbeitswirtschaft und Organisation IAO, Stuttgart

4. Funk M, Kosch T, Schmidt A (2016) Interactive worker assistance. In: Lukowicz P, Krüger A, Bulling A, Lim YK, Patel SN (eds) Proceedings of the 2016 ACM international joint conference on pervasive and ubiquitous computing. ACM, New York, pp 934-939

5. Blattgerste J, Strenge B, Renner P, Pfeiffer T, Essig K (2017) Comparing conventional and augmented reality instructions for manual assembly tasks. In: Proceedings of the 10th international conference on pervasive technologies related to assistive environments, pp 75-82

6. Uva AE, Gattullo M, Manghisi VM, Spagnulo D, Cascella GL, Fiorentino M (2018) Evaluating the effectiveness of spatial augmented reality in smart manufacturing: a solution for manual working stations. Int J Adv Manuf Technol 94(1-4):509-521

7. Hou L, Wang X, Bernold L, Love PED (2013) Using animated augmented reality to cognitively guide assembly. J Comput Civ Eng 27(5):439-451

8. Aehnelt M (2017) Informationsassistenz zur kognitiven Automatisierung manueller Montagearbeitsplätze. Dissertation, Universität Rostock, Rostock 
9. Jeske T, Meyer F, Schlick CM (2014) Einfluss der Gestaltung von Arbeitsplänen auf die Anlernzeit sensumotorischer Tätigkeiten. Zeitschrift für Arbeitswissenschaft 68(1):1-6

10. Watson G, Curran R, Butterfield J, Craig C (2008) The effect of using animated work instructions over text and static graphics when performing a small scale engineering assembly. In: Curran R, Chou S-Y, Trappey A (eds) Collaborative product and service life cycle management for a sustainable world. Springer, London, pp 541-550

11. Pustka D, Huber M, Waechter C, Echtler F, Keitler P, Newman J, Schmalstieg D, Klinker G (2011) Ubitrrack: automatic configuration of pervasive sensor networks for augmented reality. IEEE Pervas Comput 10:68-79

12. Bertram P, Birtel M, Quint F, Ruskowski M (2018) Intelligent manual working station through assistive systems. In: IFACPapersOnLine, ISSN 2405-8963, International Federation of Automatic Control, Bergamo, Italy, vol 51, issue 11, pp 170-175

13. Johansson PEC, Malmsköld L, Fast-Berglund $\AA$, Moestam L (2018) Enhancing future assembly information systems-putting theory into practice. Procedia Manuf 17:491-498

14. Hold P, Ranz F, Sihn W (2016) Konzeption eines MTM-basierten Bewertungsmodells für digitalen Assistenzbedarf in der cyberphysischen Montage. In: Schlick C (ed) Megatrend Digitalisierung: Potenziale der Arbeits- und Betriebsorganisation. GITO, Berlin, pp 295-322

15. Bornewasser M, Bläsing D, Hinrichsen S (2018) Informatorische Assistenzsysteme in der manuellen Montage: Ein nützliches Werkzeug zur Reduktion mentaler Beanspruchung? Zeitschrift für Arbeitswissenschaft 72(4):264-275
16. Keller Thimo, Bayer Christian, Bausch Phillip, Metternich Joachim (2019) Benefit evaluation of digital assistance systems for assembly workstations. Procedia CIRP 81:441-446

17. Sauermann J (2016) Performance measures and worker productivity. Choosing the right performance measures can inform and improve decision-making in policy and management. IZA World of Labor

18. Nakajima S (1995): Management der Produktionseinrichtungen (Total Productive Maintenance), Campus Verlag

19. Bokranz R, Landau K (2012) Handbuch industrial engineering. Produktivitätsmanagement mit MTM). Schäffer-Poeschel, Stuttgart

20. Minge M, Thüring M, Wagner I, Kuhr CV (2016) The meCUE questionnaire. a modular evaluation tool for measuring user experience. In: Soares M, Falcão C, Ahram TZ (eds) Advances in ergonomics modeling, usability \& special populations. Proceedings of the 7th applied human factors and ergonomics society conference 2016. Springer International Press, Switzerland, pp 115-128

21. Cohen Jacob (1988) Statistical power analysis for the behavioral sciences, 2nd edn. Erlbaum, Hillsdale

Publisher's Note Springer Nature remains neutral with regard to jurisdictional claims in published maps and institutional affiliations. 